\title{
Maternal Death Model Decreases the Expression of BDNF in Rattus Norvegicus Newborns' Cerebrum and Cerebellum
}

\author{
Duhita Dyah Apsari ${ }^{1}$, Hermanto Tri Joewono², Widjiati ${ }^{3}$ \\ ${ }^{1}$ Postgraduate Student of Reproductive Health Sciences, Faculty of Medicine, ${ }^{2}$ Department of Obstetrics \\ and Gynecology, ${ }^{3}$ Department of Embryology, Faculty of Veterinary Medicine, Airlangga University, \\ Surabaya, Indonesia
}

\begin{abstract}
Maternal death occured in the initial pregnancy period up to 42 days after giving birth. Maternal death could cause early life stress in newborns which activated the HPA axis and glucocorticoid secretion as a stress hormone marker. The developing brain was very sensitive to the initial exposure to stressors that would affected the Brain Derived Neurotrophic Factor (BDNF) expression, which was one of the most important endogenous mediators of stress responses in the brain. This study aimed to analyze BDNF expression in the cerebrum and cerebellum of Rattus norvegicus newborn with the maternal death model. The control group (K1) consisted of newborns Rattus norvegicus which were not separated from the mother until 3 days old and the treatment group (K2) is maternal death model consisted of newborns Rattus norvegicus which were separated immediately from the mother after birth until 3 days old and fed with animal milk as a substitute nutrient. After 3 days treatments, 3 newborns with the heaviest, medium, and lowest weights were taken from each mother to sacrifice. BDNF examination was carried out on cerebrum and cerebellum with imunohistochemical method and the results were analyzed with Mann Whitney test. The results showed that the mean BDNF expression in the cerebrum and cerebellum of 3 days old Rattus norvegicus with maternal death model which were separated from the mother lower than the mean BDNF expression of control group.
\end{abstract}

Keywords: BDNF expression, separation from mother, maternal death model

\section{Introduction}

Maternal death is the death of a woman during pregnancy, childbirth, or within 42 days after the end of pregnancy. Maternal death did not depend on the length and location of the pregnancy. This death could be caused by anything related to pregnancy, childbirth, or medical handling. Maternal and infant death rates were a benchmark in assessing the health status of a country. ${ }^{17,26}$

\section{Corresponding Author:}

Prof. Dr.Widjiati, DVM, M.Sc;

Department of Embryology,

Faculty of Veterinary Medicine, Airlangga University, Surabaya, Indonesia

Mulyorejo, Surabaya, East Java, Indonesia

Phone +6281330649116

Email:widjiati@fkh.unair.ac.id
Maternal death could affected the children survival, especially in the first 1,000 days of life for children up to 3 years old. The main impact of maternal death was the direct loss of the relationship between the mother and the newborn baby, causing by the absence of bonding and attachment between mother and baby and the immediate cessation of breast milk intake. Mothers also played an important role in giving and responding to communicative signals from babies after birth. The absence of bonding and attachment was one of the causes of neonatal stress. Neonatal stress is an early life stress occured in neonatal period (birth to 1 month). ${ }^{1,10}$

In early life, babies were prone to experiencing pain and stress. When a baby faced a particular stressor, the hypothalamic-pituitary-adrenal axis would be activated and the paraventricular hypothalamus secretes Corticotropin-releasing hormone ( $\mathrm{CRH})$ which then stimulated the secretion of Adrenocorticotropic hormone (ACTH) by the pituitary gland. This hormone would induce the adrenal gland cortex to secrete 
glucocorticoids (GC), namely cortisol, which was considered a stress indicator hormone. Neonatal stress in newborns was associated with cortisol hormone responses and baby behavior. Neonatal stress caused glucocorticoid increasing which could had an impact on the baby's brain development. In addition, the socioemotional stress reactivity during the early period of neonatal life would affected the brain development. ${ }^{10,11}$

The part of the brain that had cognitive function was the cerebral cortex, but recent research showed that the cerebellum contained more neurons than the cerebral cortex that allowed it to be involved in cognitive function. ${ }^{2}$ The cerebrum and cerebellum were interconnected through polysinaptics and form a system associated with cognitive function and interference neuropsychiatry. ${ }^{7}$

Brain Derived Neurotrophic Factor (BDNF) is a neurotrophic factor that played an important role in brain development because of its ability to protect brain cells from various pathological conditions. BDNF also functioned to maintain neuron survival and regulate synaptic plasticity by increasing the number of dendritic spines and synapse formation. This molecular mechanism underlies cognitive function and brain development which was influenced by BDNF regulation. ${ }^{14}$ BDNF was also one of the most important endogenous mediators of stress response in the brain. The developing brain was very sensitive to the initial exposure to stressors which would also affected BDNF expression. ${ }^{9}$ Based on this background, this study was conducted to analyze the differences in BDNF expression in the cerebrum and cerebellum of Rattus norvegicus newborns with maternal death model.

\section{Material and Method}

This research was true laboratory experimental with randomized post-test only control group design. The 3-day-old Rattus norvegicus as sample unit. Female rats were pregnant through the superovulation technique by giving a $10 \mathrm{IU}$ injection of the hormone Pregnant mare serum gonadotropin (PMSG). After 48 hours, they were given $10 \mathrm{IU}$ injection of HCG hormone and later continued to monomating. Pregnant rat diagnosis was seen at 17 hours after mating with the presence of a vaginal plug. ${ }^{25}$ The pregnant rats were cared until aterm and gave birth naturally.
Experimental animal samples were divided randomly into 2 groups, namely the control group (K1) consisting of newborn rats which were not separated from the mother until 3 days old and the treatment group (K2) is maternal death model consisting of newborn rats which were separated from the mother immediately after birth until 3 days old and fed with animal milk as a substitute nutrient. After 3 days treatments, 3 newborns with the heaviest, medium, and lowest weights were taken from each mother to be sacrificed and the head decapitation was performed. The head part was separated and then put into $10 \%$ formalin. Imonohistochemical examination of BDNF expression was carried out on histochemical cerebrum and cerebellum preparations.

\section{Findings}
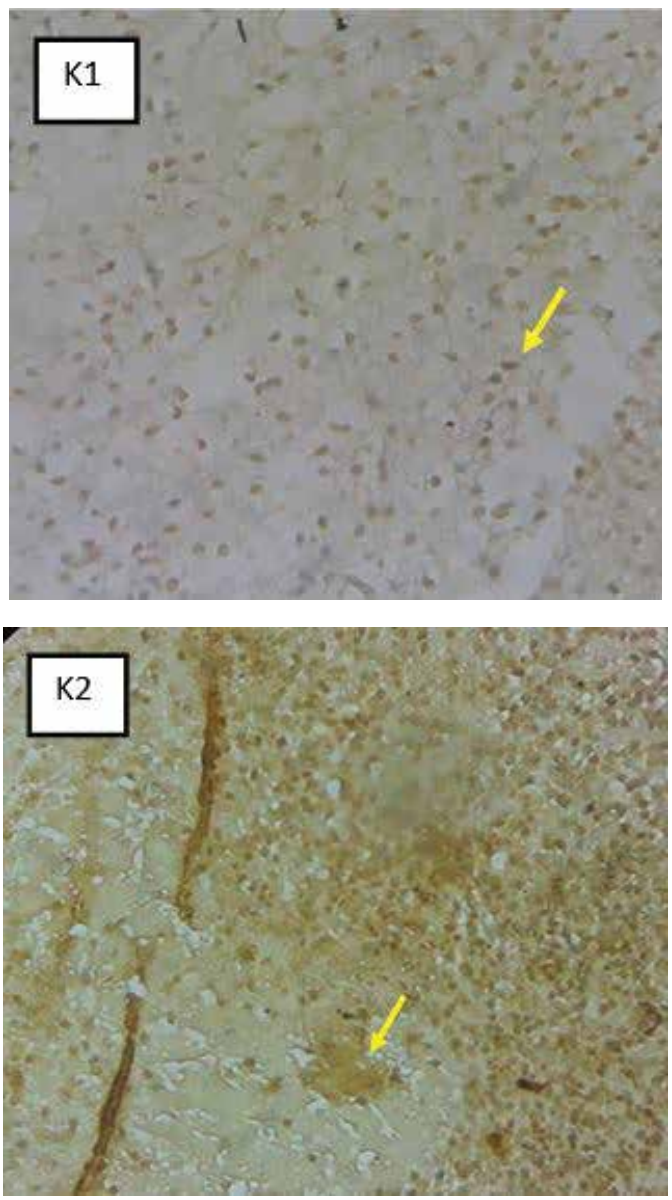

Figure 1: Histopathology of BDNF expression in the 3 days old Rattus norvegicus cerebrum between control group and treatment group. BDNF expression represented by brown color chromogen. Yellow arrow indicated the area was the maximum expression. (Immunohistochemical staining, 400x magnification; Miconos microscope MCX50LED; Optilab Plus camera) 
Table 1: Mean and standard deviation of BDNF expression cerebrum

\begin{tabular}{|c|c|c|}
\hline \multirow{2}{*}{ Group } & \multirow{2}{*}{} & BDNF Expression (IRS) \\
\cline { 3 - 3 } & & Mean \pm Standard Deviation \\
\hline $\mathrm{K} 1$ & 18 & $2.96 \pm 1.36$ \\
\hline $\mathrm{K} 2$ & 18 & $2.34 \pm 1.02$ \\
\hline
\end{tabular}

Table 2: Analysis of BDNF expression cerebrum

\begin{tabular}{|c|c|c|c|}
\hline \multicolumn{2}{|c|}{ Group } & p Value & Differences test Analysis \\
\hline K1 & K2 & $0.348^{*}$ & Mann Whitney \\
\hline
\end{tabular}

\section{Information:}

$\mathrm{K} 1=$ Newborns not separated from the mother until 3 days (control group)

$\mathrm{K} 2=$ Newborns separated from the mother until 3 days (treatment group)

*Significantly different $\mathrm{p}<0.05$
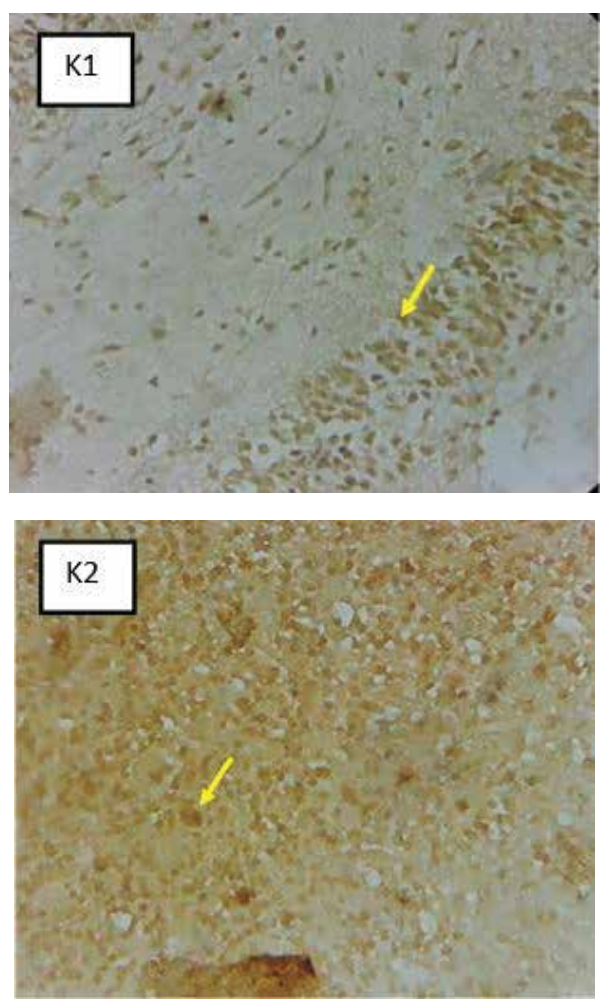

Figure 2: Histopathology of BDNF expression in the 3 days old Rattus norvegicus cerebellum between control group and treatment group. BDNF expression represented by brown color chromogen. Yellow arrow indicated the maximum expression area (immunohistochemical staining, 400x magnification; Miconos microscope MCX50LED; Optilab Plus camera)
Table 3: Mean and standard deviation of BDNF expression cerebellum

\begin{tabular}{|c|c|c|}
\hline \multirow{2}{*}{ Group } & \multirow{2}{*}{} & BDNF Expression (IRS) \\
\cline { 3 - 3 } & & Mean \pm Standard Deviation \\
\hline $\mathrm{K} 1$ & 18 & $3.06 \pm 1.48$ \\
\hline $\mathrm{K} 2$ & 18 & $2.39 \pm 1.01$ \\
\hline
\end{tabular}

Table 4: Analysis of BDNF expression cerebellum

\begin{tabular}{|c|c|c|c|}
\hline \multicolumn{2}{|c|}{ Group } & p Value & Differences Test Analysis \\
\hline K1 & K2 & $0.392^{*}$ & Mann Whitney \\
\hline
\end{tabular}

The results showed that BDNF expression mean in cerebrum of treatment group was lower than control group (Table 1) and by Mann Whitney test result showed that not significant differences in the BDNF expression in cerebrum between control group and treatment group with a $\mathrm{p}$ value $=0.348$ (significantly different need $\mathrm{p}$ value $<0.05$ ) (Table 2 ) which mean that the decrease of BDNF expression were not happened drastically based on statistical analysis. Similar results also showed in cerebellum that the BDNF expression mean of treatment group was lower than control group (Table 3) with no significant different $(\mathrm{p}$ value $=0.392)($ Table 4$)$.

\section{Discussion}

The novelty of this research is the maternal mortality model which was carried out by separation of newborns from the mother from day 0 to day 3 . Some previous research was carried out by separating newborn from the mother a few days after birth or not fully separation which mean separation only done few hours before returned back to the mother. The full separation immediately after birth and examination of BDNF cerebrum and cerebellum expression of 3 days old newborn had never been done before. The separation treatment from day 0 to day 3 early in newborn rats would be the same as the human baby separation immediately after birth from the mother for approximately 1 month. This was included in the criteria for maternal death (42 days after birth).

This research showed that BDNF expression mean of 3 days old Rattus norvegicus cerebrum and cerebellum with maternal death model was lower than control group (Table 1; Table 3). The decrease of BDNF expression could caused by several things.

In early life, babies were prone to experiencing pain and stress. Research by Shi et al (2010) states 
that chronic stress could reduce mRNA expression and BDNF protein expression in hippocampus. The study by Calabrese et al (2015) also showed that the separation of rat's pups from the mother in the postnatal day 2 to day 14 had reduced BDNF expression in the ventral hippocampus and ventromedial prefrontal cortex. However, the separation in the study was only carried out 3 hours every day then returned to the mother after the treatment. In another study, Binggio et al (2014) showed that a decrease in BDNF expression occurred significantly in the postnatal separation from the third to the 15 th day postnatal. ${ }^{4,8,21}$

Brain-Derived Neurotrophic Factor (BDNF) was a protein that played an important role in the development, maintenance, and synaptic plasticity. ${ }^{16}$ This protein was expressed in the brain, which included the frontal cortex, parietal, cingulatus, temporal, retrospenial, prirhinal, hippocampal, entorhinal cortex, brain stem, cerebrum, and cerebellum. Each area in the brain had different BDNF concentrations. ${ }^{5}$ The BDNF gene expression in the brain was influenced by many stimuli, both in physiological and pathological conditions. BDNF regulation was influenced by activation of NMDA receptors (NMDAR), calcium influx $\left(\mathrm{Ca}^{2+}\right)$, and through activation of CREB. ${ }^{10}$ BDNF was one of the most important endogenous mediators of stress response in the human and mammalian brains. The developing brain was very sensitive to the initial exposure to stressors, especially because of the reprogramming number of stress-sensitive gene pathways. ${ }^{9}$

Stressor at early of life would cause glucocorticoid to increase. Glucocorticoid played an important role in the BDNF regulation. Glucocorticoid decreased the activity of activator protein-1 (AP-1) and CREB needed in transcription of BDNF genes, influenced the cascade of BDNF signals via TrkB and p75NTR receptors, decreases the infusion of $\mathrm{Ca}^{2+}$ ions in postsynapsed membranes, inhibits trkB-mediated signal cascades, and decreases expression phospho-TrkB (pTrkB), and influence the PLC- $\gamma$ signal cascade regulation by BDNF and inhibit the PI3K-Akt pathway. ${ }^{22,23}$

With the BDNF presence, the TrkB receptor homodimerizes and initiates several signaling pathways and would support neuron survival, growth, and differentiation. Glucocorticoid(GC) passed through the plasma membrane and entered the cytosol to bind to glucocorticoid receptor (GR), and induces homodimerization (GR-GC complex). The GR-GC complex would target BDNF promoters. Increased glucocorticoid levels caused relocation of nucleus CREB regulated transcription coactivator 2 (CRTC2) to the cytosol. This could decreased the transcription activity of CREB which would caused a decrease in BDNF expression. ${ }^{12}$

In addition, regulation of BDNF expression by glucocorticoid was also related to DNA methylation and histone modification.BDNF transcripts containing exons I, II, IV, and VI were mostly expressed in transcript neurons so that they display different subcellular localization and could showed that protein was translated by different efficiency in soma or dendritic which would produce local effects. Decreasing BDNF expression was also often associated with increased DNA methylation in BDNF promoters. DNA methylation was one of the contributing factors to long-term epigenetic reprogramming and gene activity in the developing brain. Methylation of BDNF genes was often considered a key mechanism of early life stress and influenced brain function. ${ }^{13,24}$ Research by Roth et al (2009) and Blaze et al (2013) showed that early life stress in children caused changes in DNA methylation in BDNF accompanied by reduced expression in BDNF. ${ }^{6,18}$

Stress also could caused the glucocorticoid receptor translocation from the cytoplasm to the nucleus that would result in the imbalance of mitochondria and the production of NADPH oxidase (NOX). Some stress signaling pathways would follow NOX stimulation and would led to cell damage and lower BDNF expression. ${ }^{19}$

Changes in BDNF expression indicated a dependence on the time period, that was on direct or long-term effects, an acute or chronic. A recent analysis of hippocampal transcriptomies showed that the stress effects on children on BDNF expression were very dependent on age and could vary over the lifetime. ${ }^{24}$

Some of the above could explained that the maternal death model with separation from the mother could affected the BDNF expression mean, both in the cerebrum and cerebellum of 3 days old Rattus norvegicus.

\section{Conclussion}

The results showed that the BDNF expression mean of 3 days old Rattus norvegicus cerebrum and 
cerebellum with maternal death model which newborns immedietely separated from the mother after birth until 3 days was lower than the BDNF expression from control group which not separated from the mother.

Ethical Clearance: This study had obtained an ethical feasibility permit based on the Research Ethics Commission of the Faculty of Veterinary Medicine, Airlangga University.

Source of Funding: This study was self funding by authors.

Conflict of Interest: There was no conflict of interest in this study.

\section{REFERENCES}

1. Angelhoff, C., Blomqvist, Y. T., Helmer, C. S., Olsson, E., Shorey, S., Frostell, A., and Morelius, E. Effect of skin-to-skin contact on mothers' sleep quality, mood, mother-infant interaction and cortisol concentrations in neonatal care units: study protocol of a randomised controlled trial. BMJ Open Journal. 2018; 8:1-8.

2. Barton, R.A., and Venditti, C. Rapid Evolution of the Cerebellum in Humans and Other Great Apes. Current Biology. 2014; 24: 2440-2444.

3. Bekinschtein, P., Cammarota, M., Izquierdo, I., and Medina, J.H. Reviews BDNF and Memory Formation and Strage. Neuroscientist. 2008; 14(2):147-156.

4. Biggio, F., Pisu, M.G., Garau, A., Boero, G., Locci, V., Mostallino, M.C., Olla, P., Utzeri, C., and Serra, M. Maternal separation attenuates the effect of adolescent social isolation on HPA axis responsiveness in adult rats. Eur. Neuropsychopharmacol. 2014; 24.

5. Binder, D. K., and Scharfman, H. E. Brainderived Neurotrophic Factor. Growth Factors (Chur, Switzerland). 2004; 22(3): 123-131.

6. Blaze, J., Scheuing, L., and Roth, T.L. Differential methylation of genes in the medial prefrontal cortex of developing and adult rats following exposure to maltreatment or nurturing care during infancy. Dev. Neuroscience. 2013; 35: 306-316.

7. Buckner, R.L. The cerebellum and cognitive function: 25 years of insight fromanatomy and neuroimaging. Neuron, 2013; 80(3): 807-815.
8. Calabrese, F, Van der Doelen, R.H., Guidotti, G., Racagni, G., Kozicz, T., Homberg, J.R., and Riva, M.A. Exposure to early life stress regulates Bdnf expression in SERT mutant rats in an anatomically selective fashion. J. Neurochem. 2015; 132: 146-154.

9. Chen, Y., and Baram, T.Z. Toward understanding how early-life stress reprograms cognitive and emotional brain networks. Neuropsychopharmacology. 2016; 41: 197-206.

10. Chis, A., Vultura, R., Andreica, S., Prodan, A., and Miu, A.C. Behavioral and cortisol responses to stress in newborn infants: Effects of mode of delivery. Psychoneuroendocrinology. 2017; 86: 203-208.

11. Debernardo, G., Riccitelli, M., Giordano, M., Proietti, F., Sordino, D., Longini, M., Buonocore, G., and Perone, S. Rooming-in Reduces Salivary Cortisol Level of Newborn. Mediators of Inflammation. 2018.

12. Daskalakis, N.P., DeKloet, E.R., Yehuda, R., Malaspina, D., and Kranz, T.M. Early Life Stress Effects on Glucocorticoid-BDNF Inter play in the Hippocampus. Molecular Neuroscience, 2015 ; 68 .

13. Ikegame, T., Bundo, M., Murata, Y., Kasai, K., Kato, T., and Iwamoto, K. DNA methylation of the BDNF gene and its eelevance to psychiatric disorders. J. Hum. Genet. 2013; 58: 434-438.

14. Lu, B., Nagappan, G., and Lu, Y. BDNF and Synaptic Plasticity, Cognitive Function, and Dysfunction. In: Lewin G., Carter B. (eds) Neurotrophic Factors. Handbook of Experimental Pharmacology vol 220. Berlin: Springer. 2014.

15. Lyons, M.R and West, A.E. Mechanisms of specificity in neuronal activity-regulated gene transcription. Prog. Neurobiol. 2011; 94: 259-295.

16. Marosi, K., and Mattson, M. P. BDNF Mediates Adaptive Brain and Body Responses to Energetic Challenges. Trends in Endocrinology and Metabolism: TEM. 2014; 25(2): 89-98.

17. Ronsmans, C., and Graham, W.J. Maternal mortality: who, when, where, and why. The Lancet. 2006; 368(9542) : 1189-1200.

18. Roth T.L., Lubin F.D., Funk A.J., and Sweatt J.D. Lasting epigenetic influence of early-life adversity on the BDNF gene. Biol. Psychiatry. 2009; 65: 760-769. 
19. Schiavone, S., Colaianna, M., \& Curtis, L. Impact of Early Life Stress on the Pathogenesis of Mental Disorders: Relation to Brain Oxidative Stress. Current Pharmaceutical Design. 2015; 21: 14041412.

20. Scott, S., Kendali, L., Gomez, P., Howie, S. R., Zaman, S. M., Caesay, S., and Jasseh, M. Effect of maternal death on child survival in rural West Africa: 25 years of prospective surveillance data in The Gambia. PLoS ONE. 2017; 12(2): 1-14.

21. Shi, S.S., Shao, S.H., Yuan, B.P., Pan, F., and Li, Z.L. Acute Stress and Chronic Stress Change Brain-Derived Neurotrophic Factor (BDNF) and Tyrosine Kinase-Coupled Receptor (TrkB) Expression in Both Young and Aged Rat Hippocampus. Younsei Medical Journal. 2010; 51(5): 661-671

22. Suri, D., and Vaidya, V.A. Glucocorticoid regulation of brain-derived neurotrophic factor: relevance to hippocampal structural and functional plasticity. Neuroscience. 2013; 239: 196-213
23. Suri, D., Veenit, V., and Sarkar, A. Early stress evokes agedependent biphasic changes in hippocampal neurogenesis,BDNF expression, and cognition. Biol. Psychiatry. 2013; 73: 658-666

24. Suri, D., Bhattacharya, A., and Vaidya, V.A. Early stress evokes temporally distinct consequences on the hippocampal transcriptome,anxiety and cognitivebehaviour.Int.J.Neuropsychopharmacol. 2014; 17: 289-301

25. Widjiati, Dewita, Hendrawan, V. F., Purwantari, K. E., Wadji, S. A., Zulfarniansyah, A. B., and Al-Ilmi, M. F. Histopathologic Changes in Liver Tissue from Cadmium Intoxicated Mice and Treated with Curcumin during Pregnancy. Research J. Pharm. and Tech. 2018; 11(3): 863-865.

26. World Health Organization. Maternal Mortality. World Health Organization https://www.who.int/ news-room/fact-sheets/detail/maternal-mortality. 2018 . 